\title{
Synergistic Effect between Usnic Acid and Polymyxin B against Resistant Clinical Isolates of Pseudomonas aeruginosa
}

\author{
Sérgio Dias da Costa Júnior $\mathbb{D}^{1,2}$ Wagner Roberto Cirilo da Silva, ${ }^{1}$ \\ Adriana Maria Costa Marques da Silva, ${ }^{2}$ Maria Amélia Vieira Maciel, ${ }^{2}$ \\ and Isabella Macário Ferro Cavalcanti $\mathbb{D}^{1}$ \\ ${ }^{1}$ Laboratory of Immunopathology Keizo Asami, Federal University of Pernambuco, Recife, PE, Brazil \\ ${ }^{2}$ Department of Tropical Medicine, Federal University of Pernambuco, Recife, PE, Brazil \\ Correspondence should be addressed to Isabella Macário Ferro Cavalcanti; isabella.cavalcanti@ufpe.br
}

Received 29 April 2020; Accepted 21 July 2020; Published 12 August 2020

Academic Editor: Victor Kuete

Copyright (C) 2020 Sérgio Dias da Costa Júnior et al. This is an open access article distributed under the Creative Commons Attribution License, which permits unrestricted use, distribution, and reproduction in any medium, provided the original work is properly cited.

\begin{abstract}
The present study aimed to characterize the susceptibility profile of Pseudomonas aeruginosa and Acinetobacter spp. clinical isolates to polymyxin B in a public hospital in Recife-PE, Brazil, between the years of 2018 and 2019, as well as to search for the presence of the $m c r-1$ gene and evaluate the interaction between polymyxin B and usnic acid against these isolates. The strains were identified using the BD Phoenix ${ }^{\mathrm{TM}}$ automated system and the agar-spot test was used to determine the susceptibility profile to polymyxin B. The minimum inhibitory concentrations (MICs) of usnic acid and polymyxin B were determined through the broth microdilution method according to the Clinical and Laboratory Standards Institute (CLSI). Subsequently, Polymerase Chain Reaction (PCR) was performed to detect the $m c r-1$ gene in the isolates. The interaction between usnic acid and polymyxin B was evaluated by the Checkerboard assay. Among 34 isolates of P. aeruginosa, 26.5\% (9/34) were positive for the polymyxin B agar-spot test, and 11.8\% (4/ 34) presented an intermediate susceptibility $(\mathrm{MIC}=4 \mu \mathrm{g} / \mathrm{mL})$, while $14.7 \%(5 / 34)$ presented antimicrobial resistance with MIC values ranging from 8 to $32 \mu \mathrm{g} / \mathrm{mL}$. Among 38 isolates of Acinetobacter spp., 13.2\% (5/38) were positive for the polymyxin B agar-spot test and all of them were resistant to polymyxin B with a MIC value $>32 \mu \mathrm{g} / \mathrm{mL}$. The $m c r-1$ gene was not detected in the clinical isolates. Regarding usnic acid, it presented a moderate antibacterial activity against two P. aeruginosa isolates $(\mathrm{MIC}=250 \mu \mathrm{g} / \mathrm{mL})$ and no activity was detected against the others. A synergistic effect between usnic acid and polymyxin B was observed against three clinical isolates of $P$. aeruginosa which were resistant to polymyxin B $(\mathrm{FICI} \leq 0.5)$. Therefore, it was possible to observe that usnic acid is a promising candidate to be used in combination with polymyxin B against infections caused by resistant $P$. aeruginosa.
\end{abstract}

\section{Introduction}

Pseudomonas aeruginosa and Acinetobacter spp. are nonfermenting Gram-negative bacilli (NFGNB) implicated with high rates of colonization and infection in hospitalized individuals who live, in their majority, in developing countries. These microorganisms are mainly associated with cases of pneumonia and bacteremia, as well as infections related to urinary tract, wounds, and catheter. Besides, they are included in a group composed of the six most prevalent multi-resistant pathogens in nosocomial infections, known as the acronym
ESKAPE-Enterococcus faecium, Staphylococcus aureus, Klebsiella pneumoniae, Acinetobacter spp., Pseudomonas aeruginosa, and Enterobacter spp. [1,2].

The wide variety of virulence and resistance mechanisms presented by $P$. aeruginosa and Acinetobacter spp. isolates, as well as their capacity of interacting and exchanging genetic material with other bacteria, stimulates the search for resistance genes in isolates found in hospital environments [2-4]. Besides, the increasing number of infections caused by multidrug-resistant (MDR) Gram-negative strains led to the return of the polymyxins (polymyxin $\mathrm{B}$ and colistin) in medical clinics as one of the treatment options $[5,6]$. 
Polymyxins are polypeptide antibiotics which destabilize lipopolysaccharides (LPS) from the cell membranes of Gram-negative bacteria, resulting in a permeability increase and consequently in the externalization of cytoplasmatic contents and cell death [7]. However, in the past years, some Gram-negative species which were previously described as susceptible to polymyxins have been reported as resistant to this class of antimicrobials [2, 6, 8].

Studies have demonstrated that the resistance to polymyxins could be either intrinsic, such as those observed in pathogens like Brucella spp., Burkholderia cepacia complex, Edwardsiella spp., Morganella morganii, Proteus spp., Providencia spp. and Serratia spp., or acquired through chromosomal mutations. It was thereafter identified that the resistance to polymyxins was associated with a gene that could be present in mobile genetic elements, the $\mathrm{mcr}$, encoded in a plasmid from a strain of Escherichia coli in China $[5,7]$. In the following years, studies identified the presence of the $m c r-1$ gene and its variants in Acinetobacter spp. and Pseudomonas aeruginosa $[6,8]$. In addition to that, the first cases of human infections caused by $E$. coli strains harboring $m c r-1$ genes were detected in Latin America countries, such as Brazil, Argentina, and Ecuador [9-11]. Due to the increase of MDR bacilli, the scientific community has been studying combining therapies as an option to treat diseases caused by these microorganisms. Combined therapies are used in order to get a higher potency and consequently therapeutic success of a given antimicrobial. Natural compounds can act in synergy with drugs used in medical practice allowing the reduction of the required dose to achieve the cure and minimizing side effects [12-14].

Usnic acid is one of the compounds that have been studied in the past years. It is present in different species of lichens as a product of the secondary metabolism. A series of in vitro studies demonstrated not only anti-inflammatory, antioxidant, antitumor, and antiviral properties, but also a potent antibacterial activity against mycobacteria and several Gram-positive and Gram-negative bacteria [15-18]. Along with these data, the interest in this substance has risen since it could be used as a potential antimicrobial compound. The mechanism of action of usnic acid has not been fully elucidated yet. However, studies have reported that this molecule interferes with the synthesis and replication of bacterial nucleic acids [19]. Therefore, the present study aimed to characterize the susceptibility profile of $P$. aeruginosa and Acinetobacter spp. isolates to polymyxin B in a public hospital in Recife-PE, Brazil, between the years of 2018 and 2019 and also to search for the presence of the $\mathrm{mcr}$ 1 gene and evaluate the in vitro interaction between polymyxin B and usnic acid against these isolates.

\section{Material and Methods}

2.1. Study Population. The strains analyzed in this study were collected from individuals who were admitted in a public hospital in Recife-PE, Brazil. The isolates $(n=72)$ were harvested from different regions of 72 infected or colonized patients by $P$. aeruginosa and Acinetobacter spp. These isolates were provided by the Bacteriology
Department during the years of 2018 and 2019 and are part of a project approved by the Ethics Committee for Human Research of the Federal University of Pernambuco (CAE: 0490.0.172.000-11).

2.2. Clinical Isolates. The identification of the isolates was performed through the Phoenix $\mathrm{B}^{\mathrm{TM}}$ automated system. Then, the isolates were sent to the Laboratory of Bacteriology and Molecular Biology and conserved in glycerol at $-20^{\circ} \mathrm{C}$. These bacteria were reactivated in tubes containing brainheart infusion (BHI) broth (HiMedia ${ }^{\circledR}$, Mumbai, India), in which they were incubated for 48 hours at $37^{\circ} \mathrm{C}$. The bacteria were then seeded in plates containing cetrimide agar and MacConkey medium, being incubated at $35 \pm 2^{\circ} \mathrm{C}$ for 24 hours so that the purity of the colonies could be analyzed [20].

2.3. Polymyxin B Agar-Spot Test. Initially, a stock solution of $60 \mu \mathrm{g} / \mathrm{mL}$ polymyxin B sulfate salt (Sigma-Aldrich ${ }^{\circledR}$, United States) was prepared. Then, $1 \mathrm{~mL}$ of the stock solution was added to $19 \mathrm{~mL}$ of Cation-Adjusted Müeller-Hinton Broth (CAMHB) (HiMedia ${ }^{\circledR}$, Mumbai, India), corresponding to a final concentration of $3 \mu \mathrm{g} / \mathrm{mL}$, at an approximate temperature of $50^{\circ} \mathrm{C}$, being thus poured onto a sterile Petri dish. A bacterial suspension adjusted to 0.5 on the McFarland Scale was prepared to obtain a final concentration of $2-5 \times 10^{5} \mathrm{UFC} /$ well; then, it was seeded in plate as a "spot" and incubated at $35 \pm 2{ }^{\circ} \mathrm{C}$ for 20 hours. As controls, the strains Escherichia coli ATCC 25922 (polymyxin/colistin susceptible), P. aeruginosa ATCC 27853 (polymyxin/colistin susceptible), and E. coli NCTC 13846 (polymyxin/colistinresistant) were used in all plates. All samples were tested in duplicate. Reading was performed after $20 \mathrm{~h}$ according to the following interpretation: positive growth (resistant to polymyxin B) when more than 1 colony was observed; negative growth (susceptible to polymyxin B) when there were no bacterial colonies [21]. The procedures were performed in duplicate and in two independent experiments.

2.4. Broth Microdilution. In order to determine the Minimal Inhibitory Concentration (MIC), $95 \mu \mathrm{L}$ of CAMHB medium was added in each well of 96-well plates. Serial dilutions of Polymyxin B ranging from 0.06 to $32 \mu \mathrm{g} / \mathrm{mL}$ and of usnic acid ranging from 1 to $500 \mu \mathrm{g} / \mathrm{mL}$ were added. Finally, the bacterial suspensions were adjusted to 0.5 on the McFarland scale, diluted, and deposited in the wells at a final concentration of $2-5 \times 10^{5} \mathrm{UFC} /$ well. The plates were then incubated at $35 \pm 2{ }^{\circ} \mathrm{C}$ for 20 to 24 hours [20]. As controls, the strains E. coli ATCC 25922, P. aeruginosa ATCC 27853, and E. coli NCTC 13846 were used. All samples were tested in triplicate using resazurin and a viability dye. The plates were read using an ELISA plate reader at $620 \mathrm{~nm}$ wavelength (Multiskan FC, Thermo Scientific) [22]. After incubation, $25 \mu \mathrm{L}$ of resazurin was added to each well and the plates were incubated for 1 hour at $35 \pm 2^{\circ} \mathrm{C}$. When color changes were observed in the wells, where a light blue turned to a bright pink, the assay was considered positive for bacterial growth. 
MICs values were interpreted according to the CLSI Guidelines [20].

2.5. DNA Extraction. After incubating the colonies in Luria Bertani (LB) medium (HiMedia ${ }^{\circledR}$, Mumbai, India) at $35^{\circ} \mathrm{C}$ for 24 hours, the DNA extraction of the isolates was performed using the Brazol kit (LGC-Biotechnology, São Paulo, Brazil), according to the manufacturer's instructions. The extracted DNA was quantified by spectrophotometry using Nanodrop ${ }^{\mathrm{TM}} 2000 / 2000$ c (Thermo Scientific, United States), in a wavelength ranging from 260 to $280 \mathrm{~nm}$.

2.6. Polymerase Chain Reaction (PCR). The amplification reactions of the $m c r-1$ gene used the following primers: CLR5-F [5'-CGGTCAGTCCGTTTGTTC-3'] and CLR5-R [5'-CTTGGTCGGTCTGTA GGG- ${ }^{\prime}$ ], described by Liu et al. [5]. Each reaction included $10 \mathrm{ng}$ of total DNA $(1 \mu \mathrm{L})$, 10 pmol of each primer, $200 \mathrm{dNTP}, 1 \mathrm{x}$ buffer, $2.5 \mathrm{mM}$ of $\mathrm{MgCl}_{2}$, and $1 \mathrm{U}$ of Taq DNA polymerase. For each amplification, the following conditions were used: initial denaturation at $94^{\circ} \mathrm{C}$ for 5 minutes; 35 cycles of 1 minute at $94^{\circ} \mathrm{C}, 1$ minute on the annealing temperature at $53^{\circ} \mathrm{C}$, and 1 minute and 30 seconds at $72^{\circ} \mathrm{C}$; followed by a final extension of 10 minutes at $72^{\circ} \mathrm{C}[23]$. As the positive control, the DNA of the E. coli strain NCTC 13846 was included and as the negative control, ultrapure water. The PCR products were submitted to electrophoresis using a $1.5 \%$ agarose gel in $0.5 \mathrm{x}$ TBE buffer. These products were stained with blue-green (LGC-Biotechnology, São Paulo, Brazil) and visualized in an ultraviolet transilluminator and photo-documenter (Kasvi $\left.{ }^{\circledR}\right)$.

2.7. Checkerboard Method. To evaluate the interaction between usnic acid and polymyxin B (Sigma-Aldrich ${ }^{\circledR}$, United States) against polymyxin B-resistant $P$. aeruginosa clinical isolates, the Checkerboard method was performed [24]. Concentrated solutions of polymyxin B and usnic acid of $8 \times \mathrm{MIC}$ and $4 \times \mathrm{MIC}$, respectively, were used. Initially, $95 \mu \mathrm{L}$ of CAMHB was added to each well of 96-well plates. In order to obtain the final MIC and dilutions with lower values than the MIC of the respective compounds, polymyxin B was added on the $X$-axis, and usnic acid on the $Y$-axis. Finally, the bacterial concentration was adjusted to a 0.5 density on the McFarland scale to obtain the final concentration of 1 to $2 \times 10^{5} \mathrm{UFC} /$ well. Wells of column 11 were used as growth controls, containing only the culture medium and the inoculum, whereas the wells of column 12 were used as sterile controls, containing only the culture medium. The microplates were incubated at $35 \pm 2^{\circ} \mathrm{C}$ for 24 hours and after this period, reading was performed at $620 \mathrm{~nm}$.

In order to classify the interaction between the compounds, the fractional inhibitory concentration index (FICI) was calculated according to the equation $\Sigma \mathrm{FICI}=\mathrm{FICI}_{a}+$ $\mathrm{FICI}_{b}=\left(C_{a} / \mathrm{MIC}_{a}\right)+\left(C_{b} / \mathrm{MIC}_{b}\right)$, where $C_{b}$ and $C_{b}$ are the MIC values of the combined compounds and $\mathrm{MIC}_{\mathrm{a}}$ and $\mathrm{MIC}_{\mathrm{b}}$ are the MIC of the isolated compounds. The values obtained in the FICI equation were interpreted according to the classification proposed by Sopirala et al. [24]: synergistic
$(\mathrm{FICI} \leq 0.5)$, additive $(0.5>\mathrm{FICI} \leq 1.0)$, indifferent $\quad(1>$ $\mathrm{FICI} \leq 4.0)$, and antagonistic (FICI $>4.0$ ).

\section{Results and Discussion}

3.1. Clinical Isolates. Among 72 clinical isolates of NFGNB collected in a public hospital in Recife-PE, Brazil, 47.2\% (34/ 72) were identified as $P$. aeruginosa and $52.8 \%(38 / 72)$ as Acinetobacter spp. Also, $34.7 \%(25 / 72)$ of the analyzed samples were from tracheal secretion, $23.6 \%$ (17/72) from rectal swab, $15.2 \%(11 / 72)$ from urine, $13.9 \%(10 / 72)$ from blood, $8.3 \%$ (6/72) from catheter tips, and 4.16\% (3/72) from other body fluids. Similarly, studies demonstrate that samples from tracheal secretion, rectal swab, and urine of hospitalized patients present high frequencies of NFGNB related to colonization or infection (Figure 1) $[2,6,25]$.

P. aeruginosa and Acinetobacter baumannii are the two most common NFGNB in nosocomial infections. Over the last 20 years, the numbers of multidrug-resistant bacteria from these species have gradually increased around the world, especially in developing countries [25]. Deliberali et al. [25] identified NFGNB in 326 samples from patients admitted in a hospital in Porto Alegre-MG, Brazil. Among these samples, $P$. aeruginosa was identified in $65 \%$ of the isolates and A. baumannii in $16.6 \%$. The biological samples in which the higher positivity to these microorganisms was observed were tracheal aspirate (38.3\%), sputum (18.7\%), and urine $(16 \%)$.

3.2. Susceptibility Profile to Polymyxin B. The increasing number of studies which report the alarming amounts of polymyxin B-resistant Gram-negative bacteria from clinical isolates stimulates the scientific community to develop new low-cost and rapid methods that can be used for clinical diagnosis $[25,26]$. In the present study, $26.4 \%$ of 34 $P$. aeruginosa isolates (9/34) were positive for the polymyxin $\mathrm{B}$ agar-spot test presenting more than one bacterial colony, whereas among 38 isolates of Acinetobacter spp., 13.2\% (5/ 38) were positive.

Regarding the susceptibility analysis to polymyxin B using the broth microdilution assay, it was observed that $11.8 \%(4 / 34)$ of $P$. aeruginosa isolates presented an intermediate susceptibility to polymyxin B (MIC $=4 \mu \mathrm{g} / \mathrm{mL})$, whereas $14.7 \%(5 / 34)$ presented resistance to this agent $(\mathrm{MIC}=8$ to $32 \mu \mathrm{g} / \mathrm{mL})$. Among the strains of Acinetobacter spp., $13.2 \%(5 / 38)$ were resistant to polymyxin B (MIC $\geq$ $32 \mu \mathrm{g} / \mathrm{mL})$. Overall, $13.9 \%(10 / 72)$ of the analyzed isolates were resistant to polymyxin $\mathrm{B}$ (Table 1 ).

Clinical isolates of $P$. aeruginosa and Acinetobacter spp. which are resistant or have a low susceptibility to polymyxin $\mathrm{B}$ have already been reported. The polymyxins are usually the last monotherapy option to treat infections caused by P. aeruginosa and Acinetobacter spp. strains. Isolates from these resistant species are known as pan-resistant $[6,8]$.

It was possible to observe $100 \%$ of similarity between the polymyxin B agar-spot test and broth microdilution assay when the susceptibility profile of the clinical isolates was characterized. The results of the present study demonstrate 


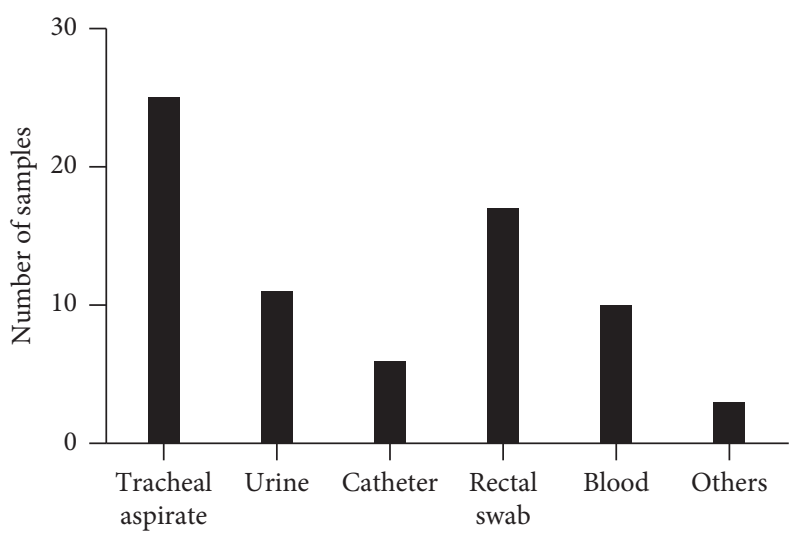

FIGURE 1: Frequency distribution of nonfermenting Gram-negative bacilli isolates in biological samples of patients from a public hospital in Recife-PE, Brazil, collected between 2018 and 2019.

TABle 1: Susceptibility profile to polymyxin B of Pseudomonas aeruginosa and Acinetobacter spp. clinical isolates.

\begin{tabular}{|c|c|c|c|}
\hline Clinical isolates & Identification of the bacteria & Polymyxin B agar-spot test & $\operatorname{MIC}(\mu \mathrm{g} / \mathrm{mL})$ \\
\hline A15 & Acinetobacter spp. & + & $>32[\mathrm{R}]$ \\
\hline A25 & Acinetobacter spp. & + & $>32[\mathrm{R}]$ \\
\hline A71 & Acinetobacter spp. & + & $>32[\mathrm{R}]$ \\
\hline A72 & Acinetobacter spp. & + & $>32[\mathrm{R}]$ \\
\hline A76 & Acinetobacter spp. & + & $>32[\mathrm{R}]$ \\
\hline PA05 & Pseudomonas aeruginosa & + & $4[\mathrm{I}]$ \\
\hline PA07 & Pseudomonas aeruginosa & + & $4[\mathrm{I}]$ \\
\hline PA18 & Pseudomonas aeruginosa & + & $8[\mathrm{R}]$ \\
\hline PA19 & Pseudomonas aeruginosa & + & $4[\mathrm{I}]$ \\
\hline PA46 & Pseudomonas aeruginosa & + & $32[\mathrm{R}]$ \\
\hline PA56 & Pseudomonas aeruginosa & + & $8[\mathrm{R}]$ \\
\hline PA57 & Pseudomonas aeruginosa & + & $32[\mathrm{R}]$ \\
\hline PA78 & Pseudomonas aeruginosa & + & $32[\mathrm{R}]$ \\
\hline PA82 & Pseudomonas aeruginosa & + & $4[\mathrm{I}]$ \\
\hline
\end{tabular}

MIC: minimum inhibitory concentration; R: resistance; I: intermediate resistance.

that the polymyxin B agar-spot test can be used as a reliable tool to screen resistant or low-susceptible $P$. aeruginosa and Acinetobacter spp. strains. Besides that, it can be easily implemented, and it has a low cost in a clinical laboratory setup [21].

Previous studies describe the benefits of using agar-spot tests with colistin or polymyxin B to screen samples and detect strains that are resistant to these antimicrobial agents. Fernandes et al. [27] screened 4620 isolates of enterobacteria using MacConkey agar supplemented with $2 \mathrm{mg} / \mathrm{L}$ colistin and confirmed the presence of resistant strains using the broth microdilution assay. Both tests were compatible regarding the susceptibility profile to colistin among the tested isolates. Therefore, the use of a method using supplemented media with polymyxins can facilitate the screening of a high number of samples [27].

Studies developed in Brazil between 2009 and 2017 reported that drugs such as the polymyxins should be the last therapeutic resource against multidrug-resistant strains of P. aeruginosa and Acinetobacter spp. Besides, pan-resistant strains of these same species have been found in clinical isolates from patients admitted in intensive care units (ICUs) in Brazilian hospitals [3, 28, 29].
Genteluci et al. [28] have reported an alarming frequency of polymyxin B-resistant strains of A. baumannii in clinical isolates from a tertiary hospital in Rio de Janeiro, Brazil. From 92 samples, 85 presented MIC values ranging from 4 to $64 \mu \mathrm{g} / \mathrm{mL}$ and were characterized as resistant to polymyxin B. Differently from Genteluci et al. [28], a study which analyzed the activities of the polymyxins B and $\mathrm{E}$ against 100 clinical isolates of $P$. aeruginosa $(n=65)$ and $A$. baumannii $(n=35)$ collected from two public hospitals from Recife-PE, Brazil, demonstrated that all isolates were sensitive to both polymyxins [2].

Grewal et al. [30] investigated the presence of NFGNB among patients admitted in a tertiary care hospital in Patiala, India, between the years of 2015 and 2016. Among 1854 culture-positive samples, 216 (11.6\%) comprised NFGNB. $P$. aeruginosa was the most common NFGNB, being isolated in 190 out of 216 samples (87.96\%), followed by A. baumannii 17/216 (7.87\%). Besides that, all isolates of $P$. aeruginosa were susceptible to polymyxin $\mathrm{B}$, whereas only $3 / 17(17.6 \%)$ of $A$. baumannii isolates were also susceptible. This data demonstrates an alarming percentage of infections caused by polymyxin B-resistant strains of A. baumannii in patients admitted in this hospital. A similar study evaluated 
the resistance profile of NFGNB present in patients admitted at the University Hospital of Nepal, between January and December 2017. Among the 1486 culture-positive cases, 402 NFGNB isolates were identified. A. baumannii was identified in $177 / 402(44 \%)$, while $P$. aeruginosa was isolated in $161 / 402(40.1 \%)$. In addition to that, all isolates of A. baumannii and $P$. aeruginosa demonstrated susceptibility to polymyxin B and colistin [31]. Studies conducted by Grewal et al. [30] and Yadav et al. [32] obtained different incidence results of polymyxin B-resistant Acinetobacter spp. and $P$. aeruginosa isolates compared to the present study. The presence of different percentages of polymyxinresistant strains in different parts of the world is related to the public health characteristics, number of inhabitants, sanitation, and economic development, those being considered determinant factors in the health-disease process [28-30, 32].

Clinical isolates of $P$. aeruginosa which presented an intermediate susceptibility to polymyxin B were present in tracheal secretion samples, whereas resistant isolates were found in urine $(3 / 5)$, catheter tip (1/5), and ulcer secretion (1/5). Polymyxin B-resistant Acinetobacter spp. isolates were found in tracheal aspirate (2/5), rectal swab (2/5), and urine $(1 / 5)$. Similarly, a study in Pakistan using NFGNB strains has described colistin-resistant isolates of $P$. aeruginosa and A. baumannii in urine, wound secretion, and blood samples [8]. Both studies demonstrate the diversified prevalence in biological samples in which strains of NFGNB resistant to polymyxins can be detected.

3.3. Presence of the mor-1 Gene. In this study, among all the polymyxin B-resistant clinical isolates, none of them presented the $m c r-1$ gene (Figure 2). This suggests that the resistance to polymyxin $\mathrm{B}$ in these microorganisms is related to different resistance mechanisms or even to a variation of the $m c r$ gene. A study performed in Pakistan investigated the susceptibility profile to colistin in 146 clinical isolates of A. baumannii $(n=62)$ and $P$. aeruginosa $(n=84)$ collected in the four biggest tertiary hospitals in Peshawar. It was verified that $9.6 \%(6 / 62)$ of A. baumannii isolates and $11.9 \%(10 / 84)$ of $P$. aeruginosa strains were resistant to colistin. The search for the $m c r-1$ gene among colistin-resistant isolates has detected one isolate of $A$. baumannii and one of $P$. aeruginosa, being the first case of $A$. baumannii and $P$. aeruginosa strains carrying the $m c r-1$ gene [8].

The resistance to polymyxin might occur through mutations or adaptation processes or during the acquisition of plasmids that carry resistance genes. However, regarding $P$. aeruginosa and Acinetobacter spp., this resistance is mainly related to complex networks involved with LPS composition or triggered by chromosomal mutations and/or differences in gene expression levels related to the LPS components. The present study did not detect the $m c r-1$ gene in NFGNB, and this fact demonstrates that these isolates might have acquired resistance through mutations or bacterial adaptation. Studies emphasize that research involving polymyxin resistance mediated by plasmids in NFGNB is needed mainly due to the constant variation in molecular epidemiology and because this gene has already been described in NFGNB isolates in other countries, such as Iraq $[8,26]$.

3.4. Usnic Acid Antibacterial Activity. During the evaluation of its antibacterial activity, it was observed that usnic acid was not effective against Acinetobacter spp. Isolates; however, it presented an inhibitory activity in all isolates of $P$. aeruginosa $(\mathrm{MIC}=250$ and $500 \mu \mathrm{g} / \mathrm{mL}$ ) (Table 2). The results obtained in the present work corroborate with the literature. In a study performed by Maciagg-Dorszyńska et al. [33], usnic acid presented antibacterial activity against Gram-positive bacteria such as Bacillus subtilis and Staphylococcus aureus; however, when evaluated for Escherichia coli, no activity was observed.

In a study conducted by Francolini et al. [31], the isomer (+)-usnic acid presented a MIC of $32 \mu \mathrm{g} / \mathrm{mL}$ against $S$. aureus, whereas for $P$. aeruginosa, its MIC was $256 \mu \mathrm{g} / \mathrm{mL}$. Ranković et al. [34] evaluated the antimicrobial activities of an acetonic extract from Usnea barbata and usnic acid purified from the same lichen species against Gram-positive microorganisms such as B. subtilis, B. mycoides, and S. aureus and the Gramnegative bacilli E. coli and K. pneumoniae. The acetonic extract and usnic acid presented MIC values of $250 \mu \mathrm{g} / \mathrm{mL}$ and $500 \mu \mathrm{g} / \mathrm{mL}$, respectively, against the Gram-positive bacteria. When evaluated against the Gram-negative bacteria, the extract and usnic acid presented MIC values of $125 \mu \mathrm{g} / \mathrm{mL}$ and $500 \mu \mathrm{g} / \mathrm{mL}$, respectively.

3.5. Synergistic Antimicrobial Activity of Polymyxin B and Usnic Acid. According to research performed on pharmacokinetics (PK), pharmacodynamics (PD), and toxicity and also from clinical trials using polymyxin $\mathrm{B}$ and colistin, these agents have a limited clinical utility. Data regarding $\mathrm{PK}$ and PD report a bactericidal concentration around $2 \mu \mathrm{g} / \mathrm{mL}$. However, most patients with renal insufficiency cannot reach this dosage, and the use of these antimicrobials is also associated with nephrotoxicity. Two randomized clinical trials have evaluated the mortality rate in patients infected by Gram-negative bacteria resistant to carbapenems and identified a $30 \%$ to $40 \%$ mortality associated with colistin in 28 days of treatment, whereas the mortality rates associated with the use of imipenem-relebactam and plazomicin were 10 and $12 \%$, respectively $[35,36]$. Therefore, the need for studies regarding alternative treatments is urgent $[2,4,37]$.

The emergence of infections caused by resistant bacteria has encouraged the use of combined therapeutic approaches in the medical clinic. The results of the combined use of piperacillin-tazobactam associated with tobramycin, as well as ceftazidime with tobramycin, had satisfactory rates of synergistic activity against MDR $P$. aeruginosa strains. In recent years, the use of cephalosporins associated with $\beta$-lactamase inhibitors has evolved with the emergence of new options for $\beta$-lactamase inhibitors. Ceftolozane-tazobactam and ceftazidime-avibactam were introduced as therapeutic options for the treatment of infections caused by MDR and extensively drug-resistant (XDR) $P$. aeruginosa, but these antibiotics presented a low 


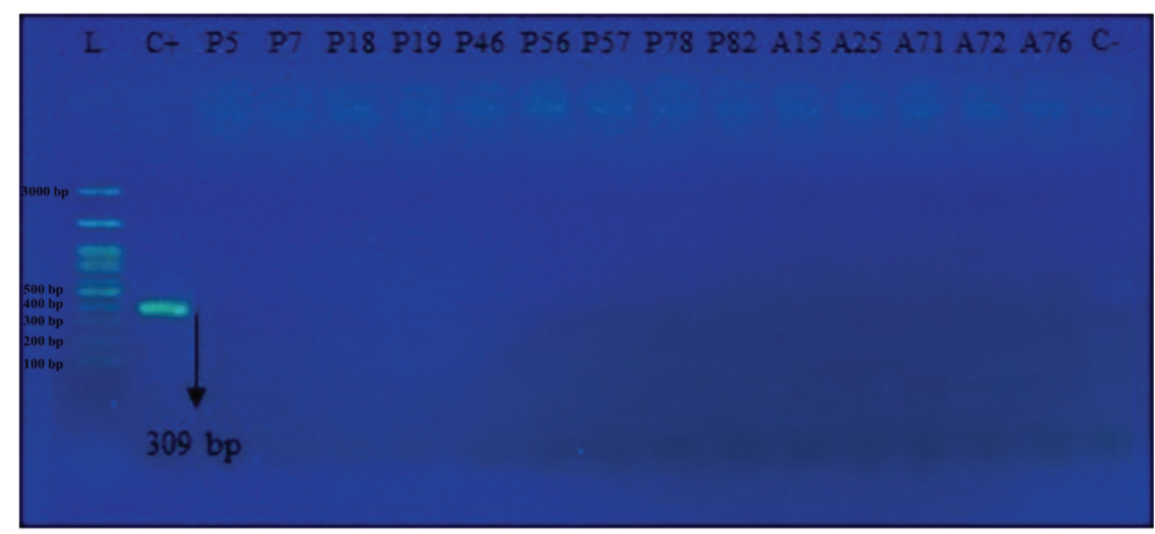

FIgURE 2: Electrophoresis of the mcr-1 PCR product in Pseudomonas aeruginosa and Acinetobacter spp. isolates obtained in a public hospital from Recife-PE, Brazil, collected between 2018 and 2019. L: molecular weight marker; C+: positive control (E. coli strain NCTC 13846); C-: negative control; bp: base pairs.

Table 2: Antimicrobial activity of usnic acid against clinical isolates of Acinetobacter baumannii and Pseudomonas aeruginosa.

\begin{tabular}{lcc}
\hline Clinical isolates & Identification of the bacteria & MIC of usnic acid $(\mu \mathrm{g} / \mathrm{mL})$ \\
\hline A15 & Acinetobacter spp. & $>500$ \\
A25 & Acinetobacter spp. & $>500$ \\
A71 & Acinetobacter spp. & $>500$ \\
A72 & Acinetobacter spp. & $>500$ \\
A76 & Acinetobacter spp. & $>500$ \\
PA05 & Pseudomonas aeruginosa & 500 \\
PA07 & Pseudomonas aeruginosa & 500 \\
PA18 & Pseudomonas aeruginosa & 500 \\
PA19 & Pseudomonas aeruginosa & 500 \\
PA46 & Pseudomonas aeruginosa & 250 \\
PA56 & Pseudomonas aeruginosa & 250 \\
PA57 & Pseudomonas aeruginosa & 500 \\
PA78 & Pseudomonas aeruginosa & 500 \\
PA82 & Pseudomonas aeruginosa & 250 \\
\hline
\end{tabular}

MIC: minimum inhibitory concentration.

inhibitory activity against metallo- $\beta$-lactamase (MBL)-producing $P$. aeruginosa. The use of aztreonam-avibactam, on the other hand, had a satisfactory activity when evaluated against enterobacteria and MBL-producing $P$. aeruginosa. Recently, the use of minocycline and doxycycline has been indicated for infections caused by MDR or XDR Acinetobacter spp. [38, 39].

In the present study, the association between polymyxin $\mathrm{B}$ and usnic acid against polymyxin-resistant $P$. aeruginosa clinical isolates was evaluated through the Checkerboard method since usnic acid alone did not present any antibacterial activity against $A$. baumannii isolates (MIC > $500 \mu \mathrm{g} / \mathrm{mL}$ ). The FICI observed in the association between usnic acid and polymyxin B varied between 0.0625 and 0.156 , being classified as synergistic for all polymyxin B-resistant $P$. aeruginosa isolates $(\mathrm{FICI} \leq 0.5)$ (Table 3 ). According to a study from Ferraz-Carvalho et al. [15], the combination of usnic acid with rifampicin has been synergistic against Mycobacterium tuberculosis isolates. Therefore, due to its recognized ability to increase the therapeutic potential of commercial drugs, usnic acid has been used as a promising alternative against resistant clinical isolates.
There are limitations regarding the use of usnic acid in in vivo models of systemic infections, due to its low aqueous solubility and hepatotoxicity after systemic administration [40]. Therefore, investigations on the therapeutic use of usnic acid in vivo have been focused on the topical application of this compound, especially in the treatment of infected wounds $[19,40,41]$. The study conducted by Zhang et al. [41] demonstrated through histopathological analyses that usnic acid had a healing effect in rats after its topical application. In this study, the following was observed: decrease in inflammatory cells, vascular regeneration, increase of fibroblast proliferation, early re-epithelialization, and epidermal keratinization.

An alternative strategy to reduce usnic acid's side effects is its encapsulation into drug delivery systems [40]. Some studies have already proposed the encapsulation of the acid into liposomes and evaluated its potential in in vitro experiments. Research conducted by Nunes et al. [42] investigated the possible curative effects of Lipo-usnic acid and gelatin-based films on epidermis wounds caused by burns using an in vivo swine model. After treatment with Lipo- 
TABLE 3: Evaluation of usnic acid and polymyxin B interaction by the Checkerboard method.

\begin{tabular}{|c|c|c|c|c|c|}
\hline \multirow{2}{*}{ Clinical isolates } & \multicolumn{2}{|c|}{ MIC $(\mu \mathrm{g} / \mathrm{mL})$} & \multirow{2}{*}{$\begin{array}{c}\text { MIC of the combination }(\mu \mathrm{g} / \mathrm{mL}) \\
\text { Polymyxin } \mathrm{B}+\text { usnic acid }\end{array}$} & \multirow{2}{*}{ FICI } & \multirow{2}{*}{ Interaction } \\
\hline & Polymyxin B & Usnic acid & & & \\
\hline PA05 & 4 & 500 & $0.25 / 15.625$ & 0.094 & Synergism \\
\hline PA07 & 4 & 500 & $0.25 / 15.625$ & 0.094 & Synergism \\
\hline PA18 & 8 & 500 & $0.25 / 31.25$ & 0.094 & Synergism \\
\hline PA19 & 4 & 500 & $0.5 / 15.625$ & 0.156 & Synergism \\
\hline PA46 & 32 & 250 & $0.25 / 7.8125$ & 0.039 & Synergism \\
\hline PA56 & 8 & 250 & $0.25 / 31.25$ & 0.094 & Synergism \\
\hline PA57 & 32 & 500 & $0.25 / 15.625$ & 0.039 & Synergism \\
\hline PA78 & 32 & 500 & $0.25 / 15.625$ & 0.039 & Synergism \\
\hline PA82 & 4 & 250 & $0.25 / 15.625$ & 0.125 & Synergism \\
\hline
\end{tabular}

MIC: minimum inhibitory concentration; FICI: fractional inhibitory concentration index.

usnic acid, tissue analyses have demonstrated the development and maturation of granulation tissue and repair of scars with the presence of satisfactory collagen deposition. The suggested mechanism of action of Lipo-usnic acid might be through its ability of promoting cellular motility. In addition, there was no presence of bacterial infections in these animal wounds [42]. However, no in vivo studies investigating the activity of usnic acid or usnic acid encapsulated in liposomes in systemic infections have been carried out yet. We also emphasize the importance of our results for future in vivo studies that aim to investigate the interaction between usnic acid and antibiotics, since the in vitro doses of these drugs are reduced and may reflect the possibility of using this combination in systemic studies.

On synergistic combinations, the involved compounds can act under different pharmacological targets neutralizing resistance mechanisms and eliminating side effects, also interacting with each other increasing their solubility and bioavailability [43]. Only a few studies suggest the possible antibacterial mechanism of action of usnic acid [19]. Gupta et al. [44] have demonstrated that usnic acid acts against S. aureus by increasing its membrane permeability leading to cell lysis. Maciąg-Dorszyńska et al. [33] verified that usnic acid can also cause the inhibition of RNA and DNA synthesis in some bacteria. In a more recent study, Sinha et al. [45] demonstrated that usnic acid acts in synergy with norfloxacin against $S$. aureus by inhibiting efflux pumps, inducing oxidative stress, inhibiting fatty acids and peptidoglycans production, altering thus the membrane potential and the metabolic activity of the bacteria destabilizing its cellular membrane.

Therefore, it is suggested that usnic acid favors the destabilization of the bacterial membrane in the presence of polymyxin $\mathrm{B}$, intensifying the electrostatic interaction with lipid A. Currently, only a few antimicrobial agents are under development; what causes concern the scientific community is the emergence of resistance mechanisms that are being constantly reported around the world. Thus, research on new therapies is of extreme importance especially regarding resistant strains of $P$. aeruginosa, for this microorganism is the most commonly involved pathogen in infected burns and wounds, as well as in systemic infections of hospitalized patients $[3,13]$.

The perspectives of this study include performing in vivo experiments to evaluate the combination between usnic acid and polymyxin B for the treatment of wound infections, and other superficial or systemic infections caused by polymyxin B-resistant $P$. aeruginosa, as well as the encapsulation of usnic acid into liposomes and the evaluation of the in vivo antibacterial activity of this Lipo-usnic acid with polymyxin B.

\section{Conclusion}

In the present study, it was possible to detect an alarming frequency of polymyxin B-resistant strains of $P$. aeruginosa and Acinetobacter spp. in patients from a public hospital in Recife-PE, Brazil. Besides, until now, no strains of these microorganisms harboring the $\mathrm{mcr}$ gene have been reported in Brazil. Thus, we reinforce the importance of local studies including phenotypic characterization and molecular epidemiology in different time intervals. Through these studies, nosocomial infections can be monitored in different hospital units, avoiding the dissemination of pathogenic microorganisms with resistant phenotypes. With that, the scientific community will be aware of the need of new therapeutic options and the rational use of antimicrobial agents, therefore assisting in the fight against bacterial resistance. The importance of detecting new genes and variants of the $m c r$ gene, as well as the construction of dissemination data on resistant NFGNB, is also highlighted. The data analysis regarding the antibacterial activity of usnic acid as a monotherapy demonstrated that this agent is weak or moderately effective against clinical isolates of Pseudomonas aeruginosa and Acinetobacter spp. However, in combination with polymyxin $\mathrm{B}$, usnic acid had a synergistic effect against polymyxin B-resistant $P$. aeruginosa clinical isolates. Therefore, usnic acid is a promising candidate against infections caused by resistant $P$. aeruginosa strains when combined with polymyxin $\mathrm{B}$.

\section{Data Availability}

The data of the present study can be acquired by contacting the corresponding author.

\section{Conflicts of Interest}

All authors declare that they have no conflicts of interest. 


\section{Authors' Contributions}

Sérgio Dias da Costa Júnior designed the project, executed the laboratorial methodology, analyzed the data, and wrote the article. Wagner Roberto Cirilo da Silva assisted in the laboratory experiments, data analysis, and writing the manuscript. Adriana Maria Costa Marques da Silva performed the bacterial DNA extractions. Maria Amélia Vieira Maciel and Isabella Macário Ferro Cavalcanti supervised the laboratory experiments and contributed to the critic evaluation of the manuscript. All the authors have read the manuscript and approved its submission.

\section{Acknowledgments}

The authors thank the Fundação de Amparo a Ciência e Tecnologia de Pernambuco (FACEPE) (APQ-0814-4.03/17) for part of the financial support of this work.

\section{References}

[1] A. Lupo, M. Haenni, and J.-Y. Madec, "Antimicrobial resistance in acinetobacter spp. and Pseudomonas spp," Microbiology Spectrum, vol. 6, no. 3, 2018.

[2] A. V. A. Lima, S. M. d. Silva, J. A. A. d. Nascimento Júnior et al., "Occurrence and diversity of intra- and interhospital drug-resistant and biofilm-forming acinetobacter baumannii and Pseudomonas aeruginosa," Microbial Drug Resistance, vol. 26, no. 7, pp. 802-814, 2020.

[3] V. V. d. Silva Júnior, L. D. Ferreira, L. R. Alves et al., "Detection of multidrug-resistant Pseudomonas aeruginosa harboring bla GES-1 and bla GES-11 in Recife, Brazil," Revista da Sociedade Brasileira de Medicina Tropical, vol. 50, no. 6, pp. 764-768, 2017.

[4] J. N. da Paz Pereira, C. A. d. N. de Andrade, J. L. da Costa Lima, R. G. de Lima Neto, P. S. R. de Araújo, and M. A. V. Maciel, "Clonal dissemination of clinical isolates of acinetobacter baumannii carriers of $16 \mathrm{~S}$ rRNA methylase genes in an oncological hospital in Recife, Brazil," Current Microbiology, vol. 77, no. 1, pp. 32-39, 2020.

[5] Y.-Y. Liu, Y. Wang, T. R. Walsh et al., "Emergence of plasmidmediated colistin resistance mechanism MCR-1 in animals and human beings in China: a microbiological and molecular biological study," The Lancet Infectious Diseases, vol. 16, no. 2, pp. 161-168, 2016.

[6] E. Caselli, M. D’Accolti, I. Soffritti, M. Piffanelli, and S. Mazzacane, "Spread of mcr-1-driven colistin resistance on hospital surfaces, Italy," Emerging Infectious Diseases, vol. 24, no. 9, pp. 1752-1753, 2018.

[7] M. E. Falagas, S. K. Kasiakou, and L. D. Saravolatz, "Colistin: the revival of polymyxins for the management of multidrugresistant gram-negative bacterial infections," Clinical Infectious Diseases, vol. 40, no. 9, pp. 1333-1341, 2005.

[8] F. Hameed, M. A. Khan, H. Muhammad et al., "Plasmidmediated mcr-1 gene in Acinetobacter baumannii and Pseudomonas aeruginosa: first report from Pakistan," Revista da Sociedade Brasileira de Medicina Tropical, vol. 52, Article ID E20190237, 2019.

[9] M. Rapoport, D. Faccone, F. Pasteran et al., "First description of mcr-1-mediated colistin resistance in human infections caused by Escherichia coli in Latin America," Antimicrobial Agents and Chemotherapy, vol. 60, no. 7, pp. 4412-4413, 2016.
[10] J. Merida-Vieyra, A. De Colsa- Ranero, P. Arzate-Barbosa et al., "First clinical isolate of Escherichia coli harboring mcr-1 gene in Mexico," PLoS One, vol. 14, no. 4, Article ID e0214648, 2019.

[11] R. Papa-Ezdra, F. Grill Diaz, M. Vieytes et al., "First three Escherichia coli isolates harbouring mcr-1 in Uruguay," Journal of Global Antimicrobial Resistance, vol. 20, pp. 187190, 2020.

[12] F. Carmona and A. M. Soares Pereira, "Herbal medicines: old and new concepts, truths and misunderstandings," Revista Brasileira de Farmacognosia, vol. 23, no. 2, pp. 379-385, 2013.

[13] P. A. Fraile-Ribot, G. Cabot, X. Mulet et al., "Mechanisms leading to in vivo ceftolozane/tazobactam resistance development during the treatment of infections caused by MDR Pseudomonas aeruginosa," Journal of Antimicrobial Chemotherapy, vol. 73, no. 3, pp. 658-663, 2018.

[14] R. E. Duval, M. Grare, and B. Demoré, "Fight against antimicrobial resistance: we always need new antibacterials but for right bacteria," Molecules, vol. 24, no. 17, p. 3152, 2019.

[15] R. S. Ferraz-Carvalho, M. A. Pereira, L. A. Linhares et al., "Effects of the encapsulation of usnic acid into liposomes and interactions with antituberculous agents against multidrugresistant tuberculosis clinical isolates," Memórias do Instituto Oswaldo Cruz, vol. 111, no. 5, pp. 330-334, 2016.

[16] A. Pompilio, A. Riviello, V. Crocetta et al., "Evaluation of antibacterial and antibiofilm mechanisms by usnic acid against methicillin-resistant Staphylococcus aureus," Future Microbiology, vol. 11, no. 10, pp. 1315-1338, 2016.

[17] J. B. Moura, A. C. d. Vargas, G. V. Gouveia et al., "In vitro antimicrobial activity of the organic extract of Cladonia substellata Vainio and usnic acid against Staphylococcus spp. obtained from cats and dogs," Pesquisa Veterinária Brasileira, vol. 37, no. 4, pp. 368-378, 2017.

[18] O. A. Luzina and N. F. Salakhutdinov, "Usnic acid and its derivatives for pharmaceutical use: a patent review (2000-2017)," Expert Opinion on Therapeutic Patents, vol. 28, no. 6, pp. 477-491, 2018.

[19] A. Galanty, P. Paśko, and I. Podolak, "Enantioselective activity of usnic acid: a comprehensive review and future perspectives," Phytochemistry Reviews, vol. 18, no. 2, pp. 527-548, 2019.

[20] CLSI, Performance Standards for Antimicrobial Susceptibilly Testing, Clinical and Laboratory Standars Institute, Wayne, PA, USA, 29th edition, 2019.

[21] E. Gonzales Escalante, K. Yauri Condor, J. A. Di Conza, and G. O. Gutkind, "Phenotypic detection of plasmid-mediated colistin resistance in enterobacteriaceae," Journal of Clinical Microbiology, vol. 58, no. 3, 2019.

[22] C. H. Teh, W. A. Nazni, A. H. Nurulhusna, A. Norazah, and H. L. Lee, "Determination of antibacterial activity and minimum inhibitory concentration of larval extract of fly via resazurin-based turbidometric assay," BMC Microbiology, vol. 17 , no. 1, p. $36,2017$.

[23] L. M. Cavaco, H. Mordhorst, and R. S. Hendriksen, PCR for Plasmid-Mediated Colistin Resistance Genes: $m c r-1$ and mcr-2 (Multiplex), Protocol Optimized at National Food Institute, Copenhagen, Denmark, 2016.

[24] M. M. Sopirala, J. E. Mangino, W. A. Gebreyes et al., "Synergy testing by etest, microdilution checkerboard, and time-kill methods for pan-drug-resistant Acinetobacter baumannii," Antimicrobial Agents and Chemotherapy, vol. 54, no. 11, pp. 4678-4683, 2010.

[25] B. Deliberali, K. N. Myiamoto, C. H. D. P. Winckler Neto et al., "Prevalência de bacilos Gram-negativos não 
fermentadores de pacientes internados em Porto Alegre-RS," Jornal Brasileiro de Patologia e Medicina Laboratorial, vol. 47, no. 5, pp. 529-534, 2011.

[26] M. Lescat, L. Poirel, C. Poirel, and P. Nordmann, “A resazurin reduction-based assay for rapid detection of polymyxin resistance in Acinetobacter baumannii and Pseudomonas aeruginosa," Journal of Clinical Microbiology, vol. 57, no. 3, 2018.

[27] M. R. Fernandes, Q. Moura, L. Sartori et al., "Silent dissemination of colistin-resistant Escherichia coli in South America could contribute to the global spread of the mcr-1 gene," Eurosurveillance, vol. 21, no. 17, p. 30214, 2016.

[28] G. L. Genteluci, D. B. C. Gomes, M. J. d. Souza, K. R. Carvalho, and M. H. S. Villas-Bôas, "Emergence of polymyxin B-resistant Acinetobacter baumannii in hospitals in Rio de Janeiro," Jornal Brasileiro de Patologia e Medicina Laboratorial, vol. 52, no. 2, 2016.

[29] P. R. L. d. A. Jácome, L. R. Alves, A. T. Jácome-Júnior et al., "Detection of bla SPM-1, bla KPC, bla TEM and bla CTX-M genes in isolates of Pseudomonas aeruginosa, Acinetobacter spp. and Klebsiella spp. from cancer patients with healthcareassociated infections," Journal of Medical Microbiology, vol. 65, no. 7, pp. 658-665, 2016.

[30] U. Grewal, R. Bakshi, G. Walia, and P. Shah, "Antibiotic susceptibility profiles of non-fermenting gram-negative bacilli at a tertiary care hospital in Patiala, India," Nigerian Postgraduate Medical Journal, vol. 24, no. 2, p. 121, 2017.

[31] I. Francolini, P. Norris, A. Piozzi, G. Donelli, and P. Stoodley, "Usnic acid, a natural antimicrobial agent able to inhibit bacterial biofilm formation on polymer surfaces," Antimicrobial Agents and Chemotherapy, vol. 48, no. 11, pp. 43604365, 2004.

[32] S. K. Yadav, R. Bhujel, S. K. Mishra, S. Sharma, and J. B. Sherchand, "Emergence of multidrug-resistant nonfermentative Gram-negative bacterial infection in hospitalized patients in a tertiary care center of Nepal," BMC Research Notes, vol. 13, no. 1, p. 319, 2020.

[33] M. Maciąg-Dorszyńska, G. Węgrzyn, and B. GuzowKrzemińska, "Antibacterial activity of lichen secondary metabolite usnic acid is primarily caused by inhibition of RNA and DNA synthesis," FEMS Microbiology Letters, vol. 353, no. 1, pp. 57-62, 2014.

[34] B. Ranković, M. Kosanić, T. Stanojković, P. Vasiljević, and N. Manojlović, "Biological activities of Toninia candida and Usnea barbata together with their norstictic acid and usnic acid constituents," International Journal of Molecular Sciences, vol. 13, no. 12, pp. 14707-14722, 2012.

[35] J. A. Mckinnell, J. P. Dwyer, G. H. Talbot et al., "Plazomicin for infections caused by carbapenem-resistant enterobacteriaceae," New England Journal of Medicine, vol. 380, no. 8, pp. 791-793, 2019.

[36] J. Motsch, C. Murta de Oliveira, V. Stus et al., "RESTORE-IMI 1: a multicenter, randomized, double-blind trial comparing efficacy and safety of imipenem/relebactam vs colistin plus imipenem in patients with imipenem-nonsusceptible bacterial infections," Clinical Infectious Diseases, vol. 70, no. 9, pp. 1799-1808, 2019.

[37] M. J. Satlin, J. S. Lewis, M. P. Weinstein et al., "Clinical and Laboratory Standards Institute (CLSI) and European Committee on Antimicrobial Susceptibility Testing (EUCAST) position statements on polymyxin B and colistin clinical breakpoints," Clinical Infectious Diseases, vol. 2020, 2020.

[38] N. G. El Chakhtoura, E. Saade, A. Iovleva et al., "Therapies for multidrug resistant and extensively drug-resistant non-fermenting gram-negative bacteria causing nosocomial infections: a perilous journey toward "molecularly targeted" therapy," Expert Review of Anti-infective Therapy, vol. 16, no. 2, pp. 89-110, 2018.

[39] R. M. Humphries, J. A. Hindler, A. Wong-Beringer et al., "Activity of ceftolozane-tazobactam and ceftazidime-avibactam against beta-lactam-tesistant Pseudomonas aeruginosa isolates," Antimicrobial Agents and Cemotherapy, vol. 61, no. 12, 2017.

[40] A. Zugic, V. Tadic, and S. Savic, "Nano- and microcarriers as drug delivery systems for usnic acid: review of literature," Pharmaceutics, vol. 12, no. 2, p. 156, 2020.

[41] Z. Zhang, Y. Zheng, Y. Li et al., "The effects of sodium usnic acid by topical application on skin wound healing in rats," Biomedicine \& Pharmacotherapy, vol. 97, pp. 587-593, 2018.

[42] P. S. Nunes, A. S. Rabelo, J. C. C. d. Souza et al., "Gelatin-based membrane containing usnic acid-loaded liposome improves dermal burn healing in a porcine model," International Journal of Pharmaceutics, vol. 513, no. 1-2, pp. 473-482, 2016.

[43] S. Phitaktim, M. Chomnawang, K. Sirichaiwetchakoon, B. Dunkhunthod, G. Hobbs, and G. Eumkeb, "Synergism and the mechanism of action of the combination of $\alpha$-mangostin isolated from Garcinia mangostana L. and oxacillin against an oxacillin-resistant Staphylococcus saprophyticus," BMC Microbiology, vol. 16, no. 1, p. 195, 2016.

[44] V. K. Gupta, S. Verma, S. Gupta et al., "Membrane-damaging potential of natural L-(-)-usnic acid in Staphylococcus aureus," European Journal of Clinical Microbiology \& Infectious Diseases, vol. 31, no. 12, pp. 3375-3383, 2012.

[45] S. Sinha, V. K. Gupta, P. Kumar et al., "Usnic acid modifies MRSA drug resistance through down-regulation of proteins involved in peptidoglycan and fatty acid biosynthesis," FEBS Open Bio, vol. 9, no. 12, pp. 2025-2040, 2019. 